\title{
Numerical analysis of large deformation processes at elevated temperatures
}

\author{
A. Andrade-Campos ${ }^{\text {a,*}}$, L.F. Menezes ${ }^{\text {b }}$, F. Teixeira-Dias ${ }^{\text {a }}$ \\ a Dep. Engenharia Mecânica, Universidade de Aveiro, Campus Universitário de Santiago, 3810-193 Aveiro, Portugal \\ b Dep. Engenharia Mecânica, Universidade de Coimbra, Pólo II da Universidade de Coimbra, 3030-201 Coimbra, Portugal
}

Received 17 December 2003; received in revised form 14 December 2004; accepted 7 July 2005

\begin{abstract}
The authors describe a theoretical model and kinematic approach suitable for the numerical analysis of problems at large deformation finite strains and rotations. The presented formulations are implemented incrementally with a non-linear and rate-dependent constitutive model, adequate to the simulation of high temperature processes. The large deformation kinematic model is based on the additive decomposition of the transformation gradient and the strain rate tensor in thermoelastic and viscoplastic parts. Stress-strain relations leading to the constitutive equations governing finite deformations are formulated on an unrotated frame of reference. These relations are obtained either by polar decomposition of the transformation gradient or by the rotation increment method. Both approaches and algorithms are thoroughly compared and discussed. The flow rule is a function of the equivalent stress and the deviatoric stress tensor, of the temperature field and of a set of internal state variables. The increments of the state variables are calculated with a forward gradient time integration procedure, based on a numerical estimation of the integral of the strain rate tensor. The model was implemented in a threedimensional finite element code and tested with a set of tensile and shear tests on aluminium alloy specimens. In order to evaluate the performance of the numerical algorithms with elevated temperature processes, tests were done at temperatures ranging from room temperature to $580 \mathrm{~K}$. Numerical simulation results are compared with experimental results and shown to be in good agreement. Real material behaviour is fairly well predicted and matches the experimental results obtained with different loading conditions at different temperatures.
\end{abstract}

(c) 2005 Elsevier B.V. All rights reserved.

Keywords: Large deformations; Large rotations; Thermoelastic-viscoplastic behaviour; Finite elements; Elevated temperatures

\section{Introduction}

In the present days, the majority of metal products undergo hot-working at least during some part of their processing history. Hot-working, which includes operations such as rolling, forging, or extrusion, can be characterized by interrupted, non-isothermal, large deformations at high strain rates and elevated temperatures.

Mathematical modelling and numerical simulation techniques, often used to study the mechanical and thermal behaviour of metallic alloys, can lead to significant reductions in the cost/efficiency relation of both the design and the analysis of hot-working operations. However, in the referred conditions, the analysis of the constitutive behaviour of most metallic alloys is a relatively hard task. As an example, the kinetics of deformation of aluminium alloys is highly temperatureand rate-dependent [1]. Additionally, during such hot-working processes, and when in the presence of strain-hardening, the evolution of the internal structure of the alloy can lead to recovery, recrystallization and/or grain-growth phenomena.

\footnotetext{
${ }^{*}$ Corresponding author. Tel.: +351 234 370830; fax: +351 234370953.

E-mail address: gilac@mec.ua.pt (A. Andrade-Campos).
} 
Consequently, the development of appropriate mathematical models (and the corresponding numerical implementation) can be a highly complex task $[2,3]$. The majority of these complexities are due to the strong non-linearities of the available constitutive laws. Additionally, the coupling of both the mechanical and the thermal processes leads to an even higher degree of complexity in the mathematical modelling and numerical implementation stages.

Large deformations and rotations are often developed during most of the technological processes listed in previous paragraphs $[4,5]$. Thus, the implementation of kinematic formulations appropriate to large deformations and rotations is mandatory. These formulations should be independent of the choice of frame of reference [6]. All field and constitutive relations must be covariant, i.e., they should remain invariant when formulated either in a spatial, material or any other convenient reference configuration.

The implemented hardening model, from the infinitesimal theory of plasticity, is extended to the large strain range on the basis of the following two assumptions: (i) the mathematical formulation is based in a hypoelastic model and (ii) it is assumed that the additive decomposition of the strain rate tensor in thermoelastic and viscoplastic parts remains a valid description for the whole deformation process.

The use of a hypoelastic law to model the material behaviour is common practice both in analytical and in numerical work $[7,8]$. This is a perfectly appropriate approach when modelling metal behaviour because elastic strains remain small when compared to plastic deformations. Additionally, differences in the formulation of the elastic constitutive behaviour have little to no effect on the calculated solution [8].

Several well known approaches can be used when performing the elastic-plastic decomposition of the deformation tensor. As an example, Green and Naghdi $[9,10]$ developed an additive decomposition of the Lagrange strain tensor into elastic and plastic parts. Afterwards, McMeeking and Rice [11] and Nagtegaal and DeJong [12], among others, used an additive decomposition of the strain rate tensor, considering that the decomposition of the rate tensor is more realistic than the decomposition of the strain tensor itself [13-17].

As a consequence, the main objective of the present work is to present a temperature- and rate-dependent constitutive model suitable for the simulation of the behaviour of aluminium alloys at elevated temperatures. The model is implemented with a kinematic approach specifically developed for large deformation processes. The development and implementation of the kinematic description in a finite element program is also presented and discussed.

\section{Kinematics for large deformation continuum mechanics}

Consider the two following configurations of a generic continuum media body $\Psi$ (Fig. 1):

(i) A reference configuration $C_{0}$, corresponding to time instant $t_{0}$ and where the position of a generic material point $P$ is defined by the position vector $\mathbf{x}_{0}$.

(ii) The current configuration $C_{t}$, at time instant $t$, where the position of the same material particle is defined by the position vector $\mathbf{x}$.

Then,

$\mathbf{x}=\mathbf{x}\left(\mathbf{x}_{0}, t\right)=\mathbf{x}_{0}+\mathbf{u}\left(\mathbf{x}_{0}, t\right)$,

where $\mathbf{u}\left(\mathbf{x}_{0}, t\right)$ is the displacement vector of the material point $P$ relative to the change of configuration from $C_{0}$ to $C_{t}$. Thus, the displacement rate $\mathbf{v}$, or velocity, of the reference point $\mathbf{x}_{0}$ is the time derivative of the position vector, defined by

$$
\mathbf{v}=\dot{\mathbf{x}} \stackrel{\text { def }}{=} \frac{\partial}{\partial t} \mathbf{x}\left(\mathbf{x}_{0}, t\right)
$$

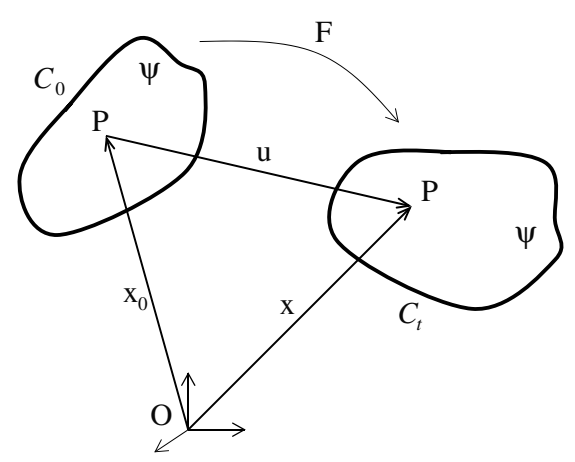

Fig. 1. Current configuration, $C_{t}$, and reference configuration, $C_{0}$, of the material at the initial and present time instants of the increment. 
For the transformation between configurations $C_{0}$ and $C_{t}$ the local strain in this material point is defined by the deformation gradient tensor $\mathbf{F}$, that is

$$
\mathbf{F}=\mathbf{F}\left(\mathbf{x}_{0}, t\right) \stackrel{\text { def }}{=} \frac{\partial}{\partial \mathbf{x}_{0}} \mathbf{x}\left(\mathbf{x}_{0}, t\right) .
$$

Relating Eq. (1) with the definition of the deformation gradient (Eq. (3)) leads to

$$
\mathbf{F}=\frac{\partial}{\partial \mathbf{x}_{0}}\left[\mathbf{x}_{0}+\mathbf{u}\left(\mathbf{x}_{0}, t\right)\right]=\mathbb{1}+\frac{\partial}{\partial \mathbf{x}_{0}} \mathbf{u}\left(\mathbf{x}_{0}, t\right),
$$

where $\mathbb{1}$ is the second order identity tensor and $\frac{\partial}{\partial \mathbf{x}_{0}} \mathbf{u}\left(\mathbf{x}_{0}, t\right)$ defines the displacement gradient.

The polar decomposition of the deformation gradient $\mathbf{F}$ can be obtained with either one of the following relations [18,19]:

$$
\mathbf{F}=\mathbf{V R}
$$

or

$$
\mathbf{F}=\mathbf{R} \mathbf{U}
$$

where $\mathbf{V}$ and $\mathbf{U}$ are the left and the right symmetric stretch tensors, respectively. Both these tensors are positive definite, i.e., $\mathbf{V}=\mathbf{V}^{\mathrm{T}}$ and $\mathbf{U}=\mathbf{U}^{\mathrm{T}}$. $\mathbf{R}$ is the orthogonal rotation tensor which, by definition, verifies the following relations:

$$
\mathbf{R}^{-1}=\mathbf{R}^{\mathrm{T}} \quad \text { and } \quad \operatorname{det}(\mathbf{R})=1 .
$$

The two polar decomposition methods, described by relations (5) and (6), are schematically illustrated in Fig. 2. An orthogonal frame of reference is defined, associated to each material point in the reference configuration $C_{0}$, such that the motion relative to this frame is only due to deformation. For the $\mathbf{R U}$ decomposition and during the transformation from $C_{0}$ to $C_{u}$ the axes are considered to be spatial and invariant with deformation. However, during the transformation from $C_{u}$ to $C_{t}$, the axes are changed to a material frame of reference in such a way that they rotate along with the body $\Psi$ at each material point. For the VR decomposition the axes follow the rotation of the transformation from $C_{0}$ to $C_{u}$. Nevertheless, during the transformation from $C_{u}$ to $C_{t}$, they are considered to be spatial axes because they are invariant with deformation.

Stress and strain tensors, and their rates, are said to be defined in the unrotated configuration $[20,21]$. The corresponding spatial velocity gradient tensor $\mathbf{L}$ is defined as

$$
\mathbf{L}(\mathbf{x}, t) \stackrel{\text { def }}{=} \frac{\partial}{\partial \mathbf{x}} \mathbf{v}(\mathbf{x}, t)=\dot{\mathbf{F}} \mathbf{F}^{-1} \text {. }
$$

The velocity gradient tensor can be decomposed in a symmetric and an antisymmetric part. The symmetric part, $\mathbf{D}$, is the strain rate tensor and the antisymmetric part, $\mathbf{W}$, is the spin rate or vorticity tensor. Both $\mathbf{D}$ and $\mathbf{W}$ are instantaneous rates, defined as

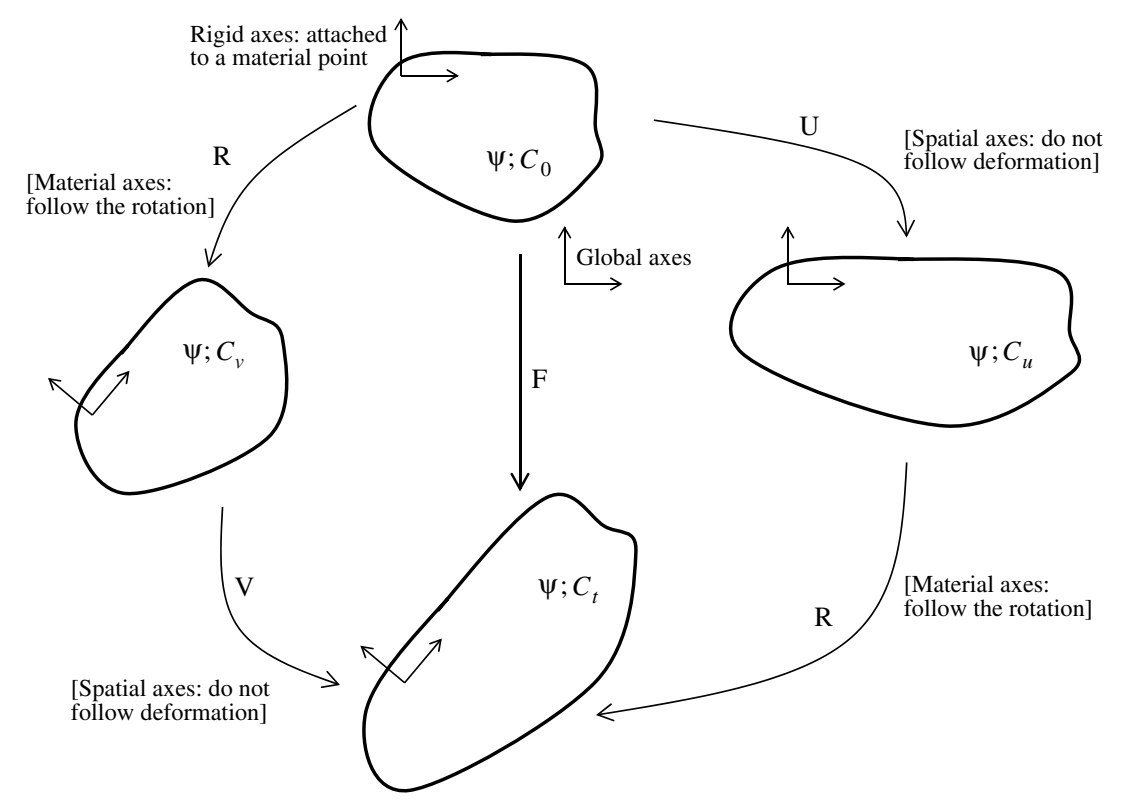

Fig. 2. Schematic representation of the two polar decomposition methods. 


$$
\mathbf{D}(\mathbf{x}, t)=\frac{1}{2}\left(\mathbf{L}+\mathbf{L}^{\mathrm{T}}\right)
$$

and

$$
\mathbf{W}(\mathbf{x}, t)=\frac{1}{2}\left(\mathbf{L}-\mathbf{L}^{\mathrm{T}}\right) .
$$

\subsection{Stress objective rates}

One of the major requirements in large deformation continuum mechanics is to achieve incremental objectivity, i.e., to maintain correct rotational transformation properties during the whole finite time step. When using an objective rate in the constitutive equations applied in a time-discretization procedure, the objectivity is achieved in small time steps. The Jaumann and the Green-Naghdi objective stress rate tensors are the ones with the widest range of application and are defined by the following expressions, respectively:

$$
\dot{\boldsymbol{\sigma}}^{J}=\dot{\boldsymbol{\sigma}}-\mathbf{W} \boldsymbol{\sigma}+\boldsymbol{\sigma} \mathbf{W}
$$

and

$$
\dot{\boldsymbol{\sigma}}^{G N}=\dot{\boldsymbol{\sigma}}-\mathbf{\Omega} \boldsymbol{\sigma}+\boldsymbol{\sigma} \boldsymbol{\Omega} .
$$

In the previous expressions, $\mathbf{W}$ is the spin tensor, already defined in Eq. (10) and $\boldsymbol{\Omega}$ is the rotation rate tensor of the body $\Psi$ $\left(\boldsymbol{\Omega}=\mathbf{R} \mathbf{R}^{\mathrm{T}}\right)$. Tensors $\mathbf{W}$ and $\boldsymbol{\Omega}$ are only identical when the principal axes of $\mathbf{D}$ coincide with the principal axes of the current stretch tensor, $\mathbf{V}$. Such is the case of either simple extension and pure rotation. However, the use of the spin tensor $\mathbf{W}$ in Eq. (11) often leads to oscillatory, and thus unrealistic, responses. These responses are predicted for the finite shear problem $[22,23]$.

In order to achieve objectivity in situations other than the small time-step discretisation, the authors reformulate the equations of the constitutive model in a co-rotational moving frame $[13,24,17]$.

\subsection{Co-rotational moving frame}

After generating a rotating frame of reference (co-rotational) and changing the frame from the fixed Cartesian axes to the generated co-rotational axes, the Cauchy stress tensor $\sigma$ becomes $[7,14,16,18]$

$$
\hat{\boldsymbol{\sigma}}=\mathbf{R}^{\mathrm{T}} \boldsymbol{\sigma} \mathbf{R} .
$$

Differentiating the previous expression relative to time $(t)$ leads to

$$
\begin{aligned}
\dot{\boldsymbol{\sigma}} & =\mathbf{R}^{\mathrm{T}}(\dot{\boldsymbol{\sigma}}-\boldsymbol{\Omega} \boldsymbol{\sigma}+\boldsymbol{\sigma} \boldsymbol{\Omega}) \mathbf{R} \\
& =\mathbf{R}^{\mathrm{T}} \boldsymbol{\sigma}^{G N} \mathbf{R} .
\end{aligned}
$$

Expression (14) states that, with a simple change of coordinates, the objective derivative becomes the time derivative of the stress tensor. This fact simplifies the whole numerical procedure. Consequently, if transformed to the unrotated configuration, the model becomes simple and evident. Additionally, the objectivity requirements are automatically verified $[24,25]$.

\section{Constitutive model}

The transformation of the strain rate tensor $\mathbf{D}$ to the corresponding unrotated strain rate tensor, $\hat{\mathbf{D}}$, yields

$$
\hat{\mathbf{D}}=\mathbf{R}^{\mathrm{T}} \mathbf{D R} .
$$

Once the kinematic transformations have eliminated the rotation effects on the rate tensors, the stress updating procedures can be the same as the conventional and simpler small-strains formulation $[14,17]$.

The constitutive model adopted by the authors is based on the following physical ideas [26,27]:

(i) Plastic deformation due to dislocation motion is inherently rate-dependent. Rate effects are due to thermal activation of various flow processes and, therefore, plastic flow is also intrinsically sensitive to temperature variations.

(ii) The instantaneous response of the material can be determined by its current state (relative to the deformation history) and is represented by a set of internal and external state variables. The evolution of these state variables is assumed to be described by rate equations. 
The following additive decomposition of the strain rate tensor $\hat{\mathbf{D}}$ is assumed, that is

$$
\hat{\mathbf{D}}=\hat{\mathbf{D}}^{\mathrm{e}}+\hat{\mathbf{D}}^{\text {th }}+\hat{\mathbf{D}}^{\mathrm{vp}}
$$

where $\hat{\mathbf{D}}^{\mathrm{e}}, \hat{\mathbf{D}}^{\text {th }}$ and $\hat{\mathbf{D}}^{\mathrm{vp}}$ are the elastic, thermal and viscoplastic parts of the strain rate tensor $\hat{\mathbf{D}}$, respectively. The thermal strain rate tensor is defined by $[28,29]$

$$
\hat{\mathbf{D}}^{\text {th }}=\alpha^{*} \dot{T} 1
$$

with

$$
\alpha^{*}=\alpha+\frac{\mathrm{d} \alpha}{\mathrm{d} T}\left(T-T_{0}\right),
$$

where $\alpha$ is the coefficient of thermal expansion (CTE) of the material, $\dot{T}$ is the temperature rate and $T_{0}$ is a reference temperature.

Bearing in mind that $\boldsymbol{\sigma}=\mathbf{C}^{\mathrm{e}}: \boldsymbol{\varepsilon}$ for thermal-hipoelastic deformation, the elastic strain rate can be defined as

$$
\hat{\mathbf{D}}^{\mathrm{e}}=\left(\hat{\mathbf{C}}^{\mathrm{e}}\right)^{-1} \dot{\hat{\boldsymbol{\sigma}}}+\dot{T} \frac{\partial\left(\hat{\mathbf{C}}^{\mathrm{e}}\right)^{-1}}{\partial T} \hat{\boldsymbol{\sigma}}
$$

where $\mathbf{C}^{\mathrm{e}}$ is the temperature-dependent elastic tensor

$$
\mathbf{C}^{\mathrm{e}}=2 \mu(T) \mathfrak{I}+\lambda(T) 1 \otimes 1 .
$$

$\mathfrak{I}$ is the fourth order identity tensor. Combining Eqs. (17)-(21), and assuming that the viscoplastic strain rate tensor is isochoric, i.e., $\operatorname{tr}\left(\hat{\mathbf{D}}^{\mathrm{vp}}\right)=0$, leads to

$$
\dot{\hat{\boldsymbol{\sigma}}}=2 \mu\left(\hat{\mathbf{D}}-\hat{\mathbf{D}}^{\mathrm{vp}}\right)+\left[\left(\kappa-\frac{2}{3} \mu\right) \operatorname{tr}(\hat{\mathbf{D}})-3 \kappa \alpha^{*} \dot{T}\right] 1-\left[2 \mu \dot{T} \frac{\partial\left(\hat{\mathbf{C}}^{\mathrm{e}}\right)^{-1}}{\partial T} \hat{\boldsymbol{\sigma}}+\left(\kappa-\frac{2}{3} \mu\right) \dot{T} \operatorname{tr}\left(\frac{\partial\left(\hat{\mathbf{C}}^{\mathrm{e}}\right)^{-1}}{\partial T} \hat{\boldsymbol{\sigma}}\right) \mathbb{1}\right],
$$

where $\kappa=\lambda+2 / 3 \mu$ is the bulk modulus. Rearranging, one obtains

$$
\dot{\hat{\boldsymbol{\sigma}}}=2 \mu\left[\hat{\mathbf{D}}-\hat{\mathbf{D}}^{\mathrm{vp}}-\dot{T} \frac{\partial\left(\hat{\mathbf{C}}^{\mathrm{e}}\right)^{-1}}{\partial T} \hat{\boldsymbol{\sigma}}\right]+\left\{\left(\kappa-\frac{2}{3} \mu\right) \operatorname{tr}\left[\hat{\mathbf{D}}+\dot{T} \frac{\partial\left(\hat{\mathbf{C}}^{\mathrm{e}}\right)^{-1}}{\partial T} \hat{\boldsymbol{\sigma}}\right]-3 \kappa \alpha^{*} \dot{T}\right\} \text {. }
$$

\subsection{Flow rule}

The constitutive equation for $\hat{\mathbf{D}}^{\mathrm{vp}}$, i.e., the flow rule, is [30,31]

$$
\hat{\mathbf{D}}^{\mathrm{vp}}=\dot{\hat{\boldsymbol{\varepsilon}}}^{\mathrm{vp}}=\frac{3 \dot{\bar{\varepsilon}}^{\mathrm{p}}}{2 \bar{\sigma}} \hat{\boldsymbol{\sigma}}^{\prime}
$$

In the previous relation, $\hat{\boldsymbol{\sigma}}^{\prime}$ is Cauchy's deviatoric stress tensor and $\bar{\sigma}$ represents the von Mises equivalent stress, defined by the following two expressions, respectively:

$$
\hat{\boldsymbol{\sigma}}^{\prime}=\hat{\boldsymbol{\sigma}}-\frac{1}{3} \operatorname{tr}(\hat{\boldsymbol{\sigma}}) \mathbb{1}
$$

and

$$
\bar{\sigma}=\sqrt{\frac{3}{2} \hat{\boldsymbol{\sigma}}^{\prime}: \hat{\boldsymbol{\sigma}}^{\prime}}
$$

The equivalent plastic strain rate, $\dot{\bar{\varepsilon}}^{\mathrm{p}}$, is considered to be dependent on

(i) The equivalent stress $\bar{\sigma}$.

(ii) The temperature $T$.

(iii) An evaluative internal state variable $s$ representing the resistance to deformation.

This dependence can be expressed, in general terms, by

$$
\dot{\bar{\varepsilon}}^{\mathrm{p}}=f(\bar{\sigma}, s, T) \text {. }
$$

The scalar parameter $s$ accounts for the microstructural state of the material and its evolution is defined by the following relation $[26,27]$ : 


$$
\begin{aligned}
\dot{s} & =g(\bar{\sigma}, s, T) \\
& =\dot{\bar{\varepsilon}}^{\mathrm{p}} h(\bar{\sigma}, s, T)-\dot{r}(s, T),
\end{aligned}
$$

where function $h(\bar{\sigma}, s, T)$ is associated to dynamic processes such as strain-hardening and dynamic recovery. Function $\dot{r}(s, T)$ accounts for static recovery phenomena. In the present work, this last function, linked to static restoration/recovery effects, was not considered [27]. In order to represent both the power law and the exponential dependence of the strain rate on the stress, the adopted relation for $\dot{\bar{\varepsilon}}^{\mathrm{p}}$ is the following [26,32]:

$$
\dot{\bar{\varepsilon}}^{\mathrm{p}}=A \exp \left(-\frac{Q}{R_{\mathrm{g}} T}\right)\left[\sinh \left(\xi \frac{\bar{\sigma}}{s}\right)\right]^{\frac{1}{m}}
$$

Additionally, the mathematical function associated to the dynamic processes is

$$
h(\bar{\sigma}, s, T)=h_{0}\left|1-\frac{s}{s^{*}}\right|^{a} \operatorname{sgn}\left(1-\frac{s}{s^{*}}\right),
$$

where $a \geqslant 1$ is a material parameter and $h_{0}$ is the athermic-hardening constant. The scalar $s^{*}$ is a saturation value of the state variable $s$ and is associated to a given temperature/strain rate pair. $s^{*}$ is defined by the relation

$$
s^{*}=\bar{s}\left[\frac{\dot{\bar{\varepsilon}}^{\mathrm{p}}}{A} \exp \left(\frac{Q}{R_{g} T}\right)\right]^{n} .
$$

In Eqs. (30)-(32), $A, Q, \xi, m, \bar{s}$ and $n$ are material parameters. Detailed procedures on the determination of these material parameters can be found, for example, in the works of Brown et al. [26] and Balasubramanian and Anand [33].

\section{Numerical implementation}

The flow rule (Eq. (24)) must be integrated over time increment $\Delta t$ [34]. At the start of the increment, state variables $\bar{\sigma}_{n}$, $s_{n}$ and $T_{n}$ are known and their corresponding values at the end of the increment $-\bar{\sigma}_{n+1}, s_{n+1}$ and $T_{n+1}-$ where $t_{n+1}=$ $t_{n}+\Delta t$, must be determined [35,36].

A forward gradient time integration procedure is implemented based on the estimation of the integration of $\hat{\mathbf{D}}^{\mathrm{vp}}$ over the current time increment $\Delta t$, in order to obtain the plastic strain increment $[27,28,30]$

$$
\Delta \hat{\boldsymbol{\varepsilon}}^{\mathrm{vp}}=\int_{t_{n}}^{t_{n+1}} \hat{\mathbf{D}}^{\mathrm{vp}} \mathrm{d} t .
$$

This tensor is obtained with the generalized mid-point rule,

$$
\Delta \hat{\boldsymbol{\varepsilon}}^{\mathrm{vp}}=\left[\hat{\mathbf{D}}_{n}^{\mathrm{vp}}+\Phi\left(\hat{\mathbf{D}}_{n+1}^{\mathrm{vp}}-\hat{\mathbf{D}}_{n}^{\mathrm{vp}}\right)\right] \Delta t
$$

where $0 \leqslant \Phi \leqslant 1$. A first order Taylor approximation is used to determine the value of $\hat{\mathbf{D}}^{\mathrm{vp}}$ at increment $n+1$, i.e.,

$$
\hat{\mathbf{D}}_{n+1}^{\mathrm{vp}}=\hat{\mathbf{D}}_{n}^{\mathrm{vp}}+\frac{\partial \hat{\mathbf{D}}_{n}^{\mathrm{vp}}}{\partial f} \Delta f+\frac{\partial \hat{\mathbf{D}}_{n}^{\mathrm{vp}}}{\partial \bar{\sigma}} \Delta \bar{\sigma}+\frac{\partial \hat{\mathbf{D}}_{n}^{\mathrm{vp}}}{\partial \boldsymbol{\sigma}^{\prime}} \Delta \boldsymbol{\sigma}^{\prime} .
$$

Considering the flow rule defined as in relation (24) then, Eq. (35) results in

$$
\hat{\mathbf{D}}_{n+1}^{\mathrm{vp}}=\frac{3 f_{n}}{2 \bar{\sigma}_{n}} \hat{\boldsymbol{\sigma}}_{n}^{\prime}+\frac{3 \hat{\boldsymbol{\sigma}}_{n}^{\prime}}{2 \bar{\sigma}_{n}} \Delta f+\frac{3}{2} f_{n} \hat{\boldsymbol{\sigma}}_{n}^{\prime}\left(-\frac{1}{\bar{\sigma}_{n}^{2}}\right) \Delta \bar{\sigma}_{n}+\frac{3 f_{n}}{2 \bar{\sigma}_{n}} \Delta \hat{\boldsymbol{\sigma}}^{\prime},
$$

where the increment of the equivalent viscoplastic strain rate, $\dot{\bar{\varepsilon}}^{\mathrm{vp}}=f(\bar{\sigma}, s, T)$, can be given by

$$
\Delta f=\frac{\partial f_{n}}{\partial \bar{\sigma}} \Delta \bar{\sigma}+\frac{\partial f_{n}}{\partial s} \Delta s+\frac{\partial f_{n}}{\partial T} \Delta T .
$$

The viscoplastic strain increment $\Delta \hat{\varepsilon}^{\mathrm{vp}}$ (Eq. (34)) becomes

$$
\Delta \hat{\varepsilon}^{\mathrm{vp}}=\Delta \bar{\varepsilon}^{\mathrm{vp}}\left(\frac{3 \hat{\boldsymbol{\sigma}}_{n}^{\prime}}{2 \bar{\sigma}_{n}}\right)+\frac{3}{2 h_{1}}\left[\Delta \hat{\boldsymbol{\sigma}}^{\prime}-\left(\hat{\boldsymbol{\sigma}}_{n}^{\prime}: \Delta \hat{\boldsymbol{\sigma}}^{\prime}\right) \frac{3 \hat{\boldsymbol{\sigma}}_{n}^{\prime}}{2 \bar{\sigma}_{n}^{2}}\right],
$$

where

$$
h_{1}=\frac{\bar{\sigma}_{n}}{\Phi f_{n} \Delta t}
$$


and

$$
\Delta \bar{\varepsilon}^{\mathrm{vp}}=\left[f_{n}+\Phi\left(\frac{\partial f_{n}}{\partial \bar{\sigma}} \Delta \bar{\sigma}+\frac{\partial f_{n}}{\partial s} \Delta s+\frac{\partial f_{n}}{\partial T}\right)\right] \Delta t .
$$

One possible procedure that can be used to update the stress tensor is to rotate the increment of spatial deformation to the unrotated configuration. In order to perform this, it is imperative to calculate the rotation tensor $\mathbf{R}$. To achieve this, two distinct methods are described, namely:

(i) The polar decomposition method [7,23,37,38], where $\mathbf{R}=\mathbf{F U}^{-1}$.

(ii) The incremental rotation tensor method, where $\mathbf{R}=\mathbf{r} \mathbf{R}_{0}\left(\mathbf{R}_{0}\right.$ is the rotation tensor in the previous time increment $)$ and $\mathbf{r}$ is the incremental rotation associated to the spin tensor $\mathbf{W}[5]$.

The use of the first method is associated to the Green-Naghdi objective stress rate. The second method is related to Jaumann objective stress rate.

The computational effort required to perform the polar decomposition should be insignificant when compared to the effort needed by the solver. Nevertheless, it should be higher than the effort needed by the second method. Additionally, despite the higher number of calculations needed, the polar decomposition method should lead to more accurate results than the incremental rotation tensor method for the reason that its theoretical bases are stronger. This conjecture is made considering that, as the incremental rotation tensor method depends on the last rotation tensor calculated, there is a possibility of accumulation of incremental error. The algorithm corresponding to the polar decomposition method can be briefly described as follows:

(1) Compute the right Cauchy-Green tensor, $\mathbf{C}$, and its square, $\mathbf{C}^{2}$, with respect to the reference configuration, as

$$
\begin{aligned}
& \mathbf{C}=\mathbf{F}^{\mathrm{T}} \mathbf{F}, \\
& \mathbf{C}^{2}=\mathbf{C}^{\mathrm{T}} \mathbf{C}=\mathbf{F F}^{\mathrm{T}} \mathbf{F}^{\mathrm{T}} \mathbf{F} .
\end{aligned}
$$

(2) Determine the eigenvalues of $\mathbf{C}: \lambda_{1}^{2}, \lambda_{2}^{2}$ and $\lambda_{3}^{2}$.

(3) Calculate the invariants of $\mathbf{U}$ and the determinant of $\mathbf{F}$ :

$$
\begin{aligned}
& \mathrm{I}_{U}=\lambda_{1}+\lambda_{2}+\lambda_{3}, \\
& \mathrm{II}_{U}=\lambda_{1} \lambda_{2}+\lambda_{2} \lambda_{3}+\lambda_{1} \lambda_{3}, \\
& \mathrm{III}_{U}=\lambda_{1} \lambda_{2} \lambda_{3}=\operatorname{det}(\mathbf{F}) .
\end{aligned}
$$

(4) Compute the inverse of $\mathbf{U}$ :

$\mathbf{U}^{-1}=\gamma_{1}\left(\gamma_{2} \mathbb{1}+\gamma_{3} \mathbf{C}+\gamma_{4} \mathbf{C}^{2}\right)$

with $\gamma_{i} \forall_{i=1, \ldots, 4}$ defined as

$$
\left\{\begin{array}{l}
\gamma_{1}=\frac{1}{\mathrm{III}_{U}\left(\mathrm{I}_{U} \mathrm{II}_{U}-\mathrm{III}_{U}\right)}, \\
\gamma_{2}=\mathrm{I}_{U} \mathrm{II}_{U}^{2}-\mathrm{III}_{U}\left(\mathrm{I}_{U}^{2}-\mathrm{II}_{U}\right), \\
\gamma_{3}=-\mathrm{III}_{U}-\mathrm{I}_{U}\left(\mathrm{I}_{U}^{2}-2 \mathrm{II}_{U}\right), \\
\gamma_{4}=\mathrm{I}_{U} .
\end{array}\right.
$$

(5) Estimate $\mathbf{R}$ through:

$$
\mathbf{R}=\mathbf{F U}^{-1} \text {. }
$$

The algorithm for the incremental rotation tensor method consists of only one step, which corresponds to updating the rotation tensor as $\mathbf{R}=\mathbf{r R}_{0}$. The procedure used to calculate the incremental rotation tensor is described in Eq. (41) [39].

$$
\mathbf{r}=1+\frac{1}{2}\left[\frac{\partial u}{\partial x}-\left(\frac{\partial u}{\partial x}\right)^{\mathrm{T}}\right]+\frac{1}{8}\left\{3 \frac{\partial u}{\partial x}-\left[\left(\frac{\partial u}{\partial x}\right)^{\mathrm{T}}\right]^{2}-\left(\frac{\partial u}{\partial x}\right)^{\mathrm{T}} \frac{\partial u}{\partial x}-\frac{\partial u}{\partial x}\left(\frac{\partial u}{\partial x}\right)^{\mathrm{T}}\right\} .
$$

Both methods, the polar decomposition and the incremental rotation tensor methods, should be performed in a mid-increment scheme, proposed by Hughes and Winget [40], in which deformation rates are evaluated on the unrotated intermediate configuration. 
Table 1

Material parameters used in the constitutive relations for the AA1050-O aluminium alloy used in the tests

\begin{tabular}{|c|c|}
\hline Parameter & Value \\
\hline$A\left(\mathrm{~s}^{-1}\right)$ & $103.3 \times 10^{3}$ \\
\hline$Q$ (at room temperature) $(\mathrm{kJ} / \mathrm{mol})$ & $165.0 \times 10^{3}$ \\
\hline$\xi$ & 6.9 \\
\hline$m$ & 0.134 \\
\hline $\bar{s}(\mathrm{MPa})$ & 34.0 \\
\hline$n$ & 0.0255 \\
\hline$h_{0}(\mathrm{MPa})$ & 595.7 \\
\hline$a$ & 1.108 \\
\hline
\end{tabular}

\section{Problem definition}

The authors performed a set of numerical simulations in order to evaluate the suitability of the model to the simulation of the behaviour of some aluminium alloys at elevated temperatures. A set of numerical simulations was also performed in order to test the numerical algorithms leading to computation of the rotation tensor $\mathbf{R}$.

All the finite element simulations were performed with a 99.5\% aluminium alloy (AA1050-O commercial alloy). This particular aluminium alloy was selected because it does not have excessive solutes and precipitates [41]. Some material properties for this alloy are temperature-dependent. The elastic modulus and the Poisson and Lamé coefficients are given as, respectively [42]:

$$
\begin{aligned}
E(T) & =72474.0-43.48(T-273)(\mathrm{MPa}), \\
v(T) & =\frac{18392.0-9.366(T-273)}{54082.0-34.114(T-273)}, \\
\mu(T) & =27000.0-17.057(T-273)(\mathrm{MPa}),
\end{aligned}
$$

where $T$ is the temperature in Kelvin.

The material parameters used in the constitutive equations (24)-(32) were adapted from experimental tests performed by the authors. This experimental data has provided preliminary values which were subsequently adapted to the problem. The constitutive parameters are listed in Table 1 [42].

For all three-dimensional simulations, a 3D hexahedric eight-node element was used. A detailed description of this finite element is not in the scope of this work but can be found, for example, in the works of Hughes [43], Pinsky et al. [44] or Zienkiewicz and Taylor [45].

The integration scheme used for this element is the one defined by Nakamura et al. [46]. The strain-displacement matrix $\mathbf{B}$ is replaced by $\mathbf{B}^{\text {stab }}$, defined by

$$
\mathbf{B}^{\text {stab }}=\mathbf{B}^{\text {dev }}+\overline{\mathbf{B}}^{\text {dil }}+\Theta\left[\mathbf{B}^{\text {dil }}-\overline{\mathbf{B}}^{\text {dil }}\right]
$$

where $\mathbf{B}^{\text {dev }}$ and $\mathbf{B}^{\text {dil }}$ are the deviatoric and dilatational parts of $\mathbf{B}$, and $\overline{\mathbf{B}}^{\text {dil }}$ is determined by Hughes's $\bar{B}$ method $[43,47]$ :

$$
\overline{\mathbf{B}}^{\text {dil }}=\frac{1}{3}\left[\begin{array}{llllll}
\overline{\mathbf{B}}_{1} & \overline{\mathbf{B}}_{1} & \overline{\mathbf{B}}_{1} & 0 & 0 & 0 \\
\overline{\mathbf{B}}_{2} & \overline{\mathbf{B}}_{2} & \overline{\mathbf{B}}_{2} & 0 & 0 & 0 \\
\overline{\mathbf{B}}_{3} & \overline{\mathbf{B}}_{3} & \overline{\mathbf{B}}_{3} & 0 & 0 & 0
\end{array}\right]^{\mathrm{T}} .
$$

The $\overline{\mathbf{B}}^{\text {dil }}$ parameters are defined by the mean dilatation method [48] where

$$
\overline{\mathbf{B}}_{i}=\frac{\partial \overline{\mathbf{N}}_{i}}{\partial x_{i}}=\frac{1}{V} \int_{V^{\mathrm{e}}} \frac{\partial \mathbf{N}_{i}}{\partial x_{i}} \mathrm{~d} V^{\mathrm{e}} .
$$

Although the use of $\overline{\mathbf{B}}$, and consequently $\overline{\mathbf{B}}^{\text {dil }}$, prevents the exhibition of locking, this procedure can introduce spurious hourglass modes. The scheme described by Eq. (45) avoids this behaviour and reduces locking without increasing the computational cost. When $\Theta=1.0$ the full integration scheme results and when $\Theta=0.0$ the $\bar{B}$ method is obtained. It is wise to use $\Theta<0.15$ to avoid locking. In the following tests, $\Theta=0.0$ was used.

\section{Experimental work}

In order to validate the proposed models, experimental work was done concerning strain-hardening in proportional loading for uniaxial tension and simple shear, at different temperatures. For this purpose, aluminium alloy sheet samples, in the H14 condition, with $1 \mathrm{~mm}$ of thickness, and whose chemical composition is given in Table 2, were used. The material 
Table 2

Chemical composition of the AA1050-O aluminium alloy (\%)

\begin{tabular}{llllllllllll}
\hline $\mathrm{Al}$ & $\mathrm{Cu}$ & $\mathrm{Fe}$ & $\mathrm{Mg}$ & $\mathrm{Mn}$ & $\mathrm{Si}$ & $\mathrm{Ti}$ & $\mathrm{V}$ & $\mathrm{Zn}$ & $\mathrm{Ga}$ & $\mathrm{Cr}$ \\
\hline 99.586 & 0.002 & 0.280 & 0.001 & 0.001 & 0.089 & 0.011 & 0.007 & 0.005 & 0.016 & 0.001 \\
\hline
\end{tabular}

was annealed at $618 \mathrm{~K}$ for $50 \mathrm{~min}$ in order to recrystallize the grain structure and obtain an average grain size under $30 \mu \mathrm{m}$. The AA1050-O sheet was deformed in uniaxial tension using specimens oriented with the rolling direction. The uniaxial specimen gauge length and width were 35 and $7.5 \mathrm{~mm}$, respectively. The cross-head speed for the uniaxial tests was $2.1 \mathrm{~mm} \mathrm{~min}^{-1}$, leading to a constant strain rate of about $\dot{\varepsilon}=0.001 \mathrm{~s}^{-1}$.

Shear tests were also performed parallel to the rolling direction, at $0^{\circ}$. The shear strain was effectively imposed in a $60 \times 3(\mathrm{~mm})$ area on $60 \times 15(\mathrm{~mm})$ specimens. The cross-head speed for the shear tests was $3.6 \mathrm{~mm} \mathrm{~min}^{-1}$. This speed was determined in order to lead to a shear strain rate of approximately $0.02 \mathrm{~s}^{-1}$.

\section{Results and discussion}

A large set of numerical simulations and experimental tests was performed in the present work. As already referred, homogeneous uniaxial tensile and shear tests were chosen in order to evaluate the capabilities of the model and the validity of the model parameters at different temperatures.

\subsection{Homogeneous uniaxial tension}

A tensile strain rate of $0.001 \mathrm{~s}^{-1}$ was used in the calculations and adiabatic conditions were assumed. The simulations were performed on a model with identical geometry to the experimental specimens referred in Section 6.

Stress-strain curves obtained in the homogeneous uniaxial tension simulations are shown in Fig. 3. As expected, uniaxial stress levels decrease with the increase of temperature and work-hardening is more accentuated for the lower temperatures.

From those results, it should be noted that the elastic portion of the stress-strain curve is extremely small, close to $0.1 \%$ of the tensile strain. Stress values are in the range of almost pure aluminium material and are lower than $120 \mathrm{MPa}$ [41], as expected. For comparison purposes, experimental results are also shown in Fig. 3. It can be seen that the numerical results obtained with the constitutive model described at Section 3.1 and with the chosen parameters are quite accurate. However, accuracy is not good in the early stages of the test. This is probably due to a deficient determination of the material parameters. Nevertheless, there is a tendency for better accuracy at the higher temperature levels, which reflects the specific nature of the constitutive law implemented. Note that this constitutive model was specifically developed for hot-working/hardening $[2,3]$.

\subsection{Shear stress tests}

Generally, homogeneous uniaxial tensile tests are not sufficient to characterize the constitutive behaviour of the material. In order to better evaluate the accuracy of the material parameters, obtained by curve-fitting procedures, already used in the uniaxial tensile tests, numerical simulations of shear tests were also performed at different temperatures.

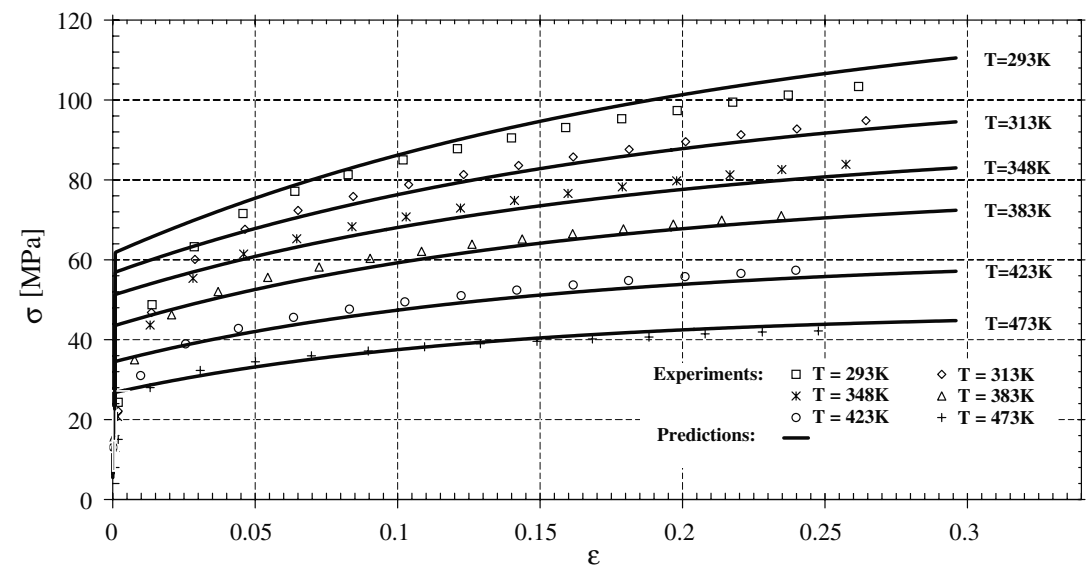

Fig. 3. Uniaxial tension stress-strain curves at different temperatures: comparison between calculated and experimental results. 


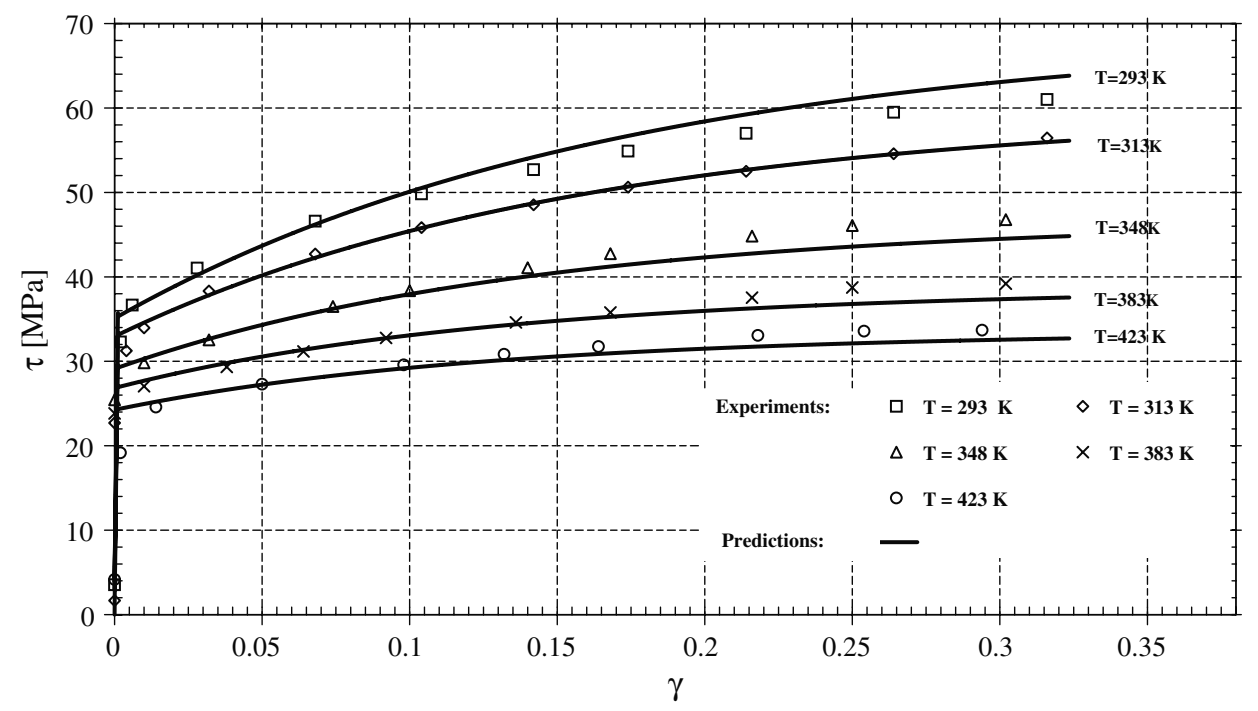

Fig. 4. Shear stress-strain curves at different temperatures: comparison between calculated and experimental results.

In order to obtain a shear strain rate of approximately $0.02 \mathrm{~s}^{-1}$, as the experimental tests, the boundary condition speed for the shear tests simulation was $3.6 \mathrm{~mm} \mathrm{~min}^{-1}$. The calculated shear stress-strain response, for several sets of temperature data, compared with corresponding experimental results, is shown in Fig. 4.

The numerical model produces shear stress-strain responses in good agreement with the experimental results. From Fig. 4 it can be seen that accuracy is good for shear deformation values under 0.15 . On the other hand, accuracy is not so good for shear deformation values over 0.2 . This fact shows that the process by which material parameters were determined should be improved.

\subsubsection{Rotation analysis}

A three-dimensional rotation can be defined in terms of the Eulerian angles $(\xi, \eta, \zeta)$. Defining ${ }^{1}$ that $\xi$ is the rotation about the $x$-axis, $\eta$ is the rotation about the new $y$-axis (after $\xi$ rotation) and $\zeta$ is the rotation about the $z$-axis (after $\xi$ and $\eta$ rotations), the rotation tensor can be easily determined as the product of three rotations, that is $[49,50]$

$$
\mathbf{R}=\mathbf{R}_{\xi} \mathbf{R}_{\eta} \mathbf{R}_{\zeta},
$$

yielding,

$$
\mathbf{R}=\left[\begin{array}{ccc}
C_{1} C_{3}-\sin (\zeta) \sin (\xi) & C_{1} \sin (\xi)+\sin (\zeta) C_{3} & -\cos (\zeta) \sin (\eta) \\
-C_{2} C_{3}-\cos (\zeta) \sin (\xi) & -C_{2} \sin (\xi)+\cos (\zeta) C_{3} & \sin (\zeta) \sin (\eta) \\
\sin (\eta) C_{3} & \sin (\eta) \sin (\xi) & \cos (\eta)
\end{array}\right]
$$

in which,

$$
C_{1}=\cos (\zeta) \cos (\eta), \quad C_{2}=\sin (\zeta) \cos (\eta), \quad C_{3}=\cos (\xi) .
$$

With the matrix defined in Eq. (49) it is possible to obtain the angles between the rotating frames and the fixed cartesian axes. The rotation tensor is either calculated by the polar decomposition method or by the rotation increment method. Subsequently, it is used in expression (49) in order to obtain the Euler angles. For the homogeneous uniaxial tension test, the use of different co-rotational frames has no influence in the response of the flow rule to the applied solicitation and, consequently, in the results. This fact can be explained due to the total absence of rotations.

The use of different co-rotational frames has a strong influence in the results whenever shear solicitations lead to strains above $80 \%$ [21,22]. Thus, the numerical rotational configuration takes a major role when large rotations occur.

Fig. 5 shows the use of the two different methods to calculate the rotation tensor in the shear test at room temperature. Both methods - the polar decomposition method and the rotation increment method-lead to the same values of the Euler angles. Angles $\xi$ and $\zeta$ have no physical meaning because, in a shear test, there is only deformation in the $O x z$ plane. The value of $\eta$ is very small because motion is almost all due to pure deformation. Thus, the frames remain almost invariant with deformation.

\footnotetext{
${ }^{1}$ Positive rotations are clockwise.
} 


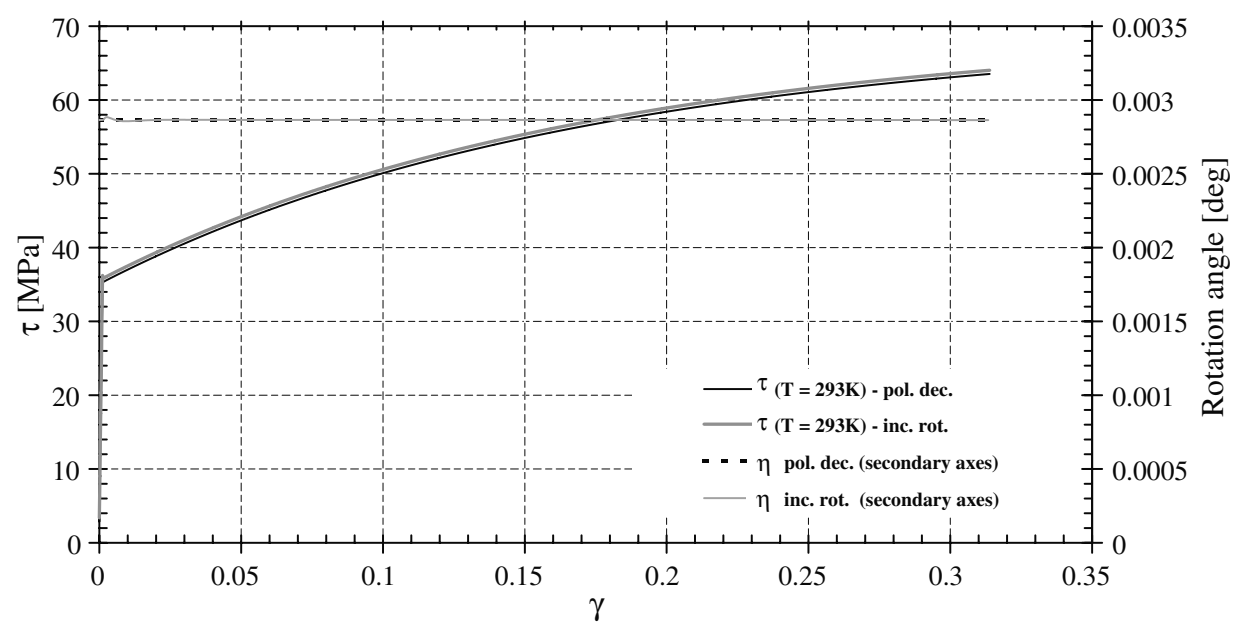

Fig. 5. Shear stress-strain curves at room temperature: comparison between the polar decomposition and the incremental rotation methods.

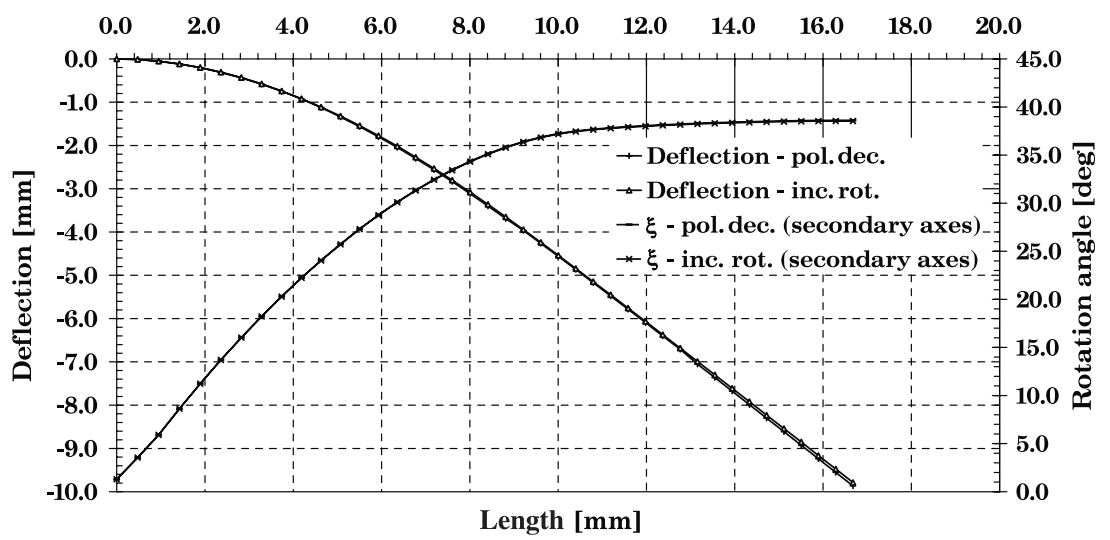

Fig. 6. Eulerian angles curves on a beam bending test at room temperature: comparison between the polar decomposition and the incremental rotation methods.

For all strain values, the use of the different methods to calculate the rotation tensor has no influence in the results.

With the bending test it is also possible to compare the accuracy of both methods, comparing the maximum beam deflexion and the calculated Eulerian angles. Fig. 6 shows the deflection and rotation results for a simple three-dimensional beam bent on its free end up to approximately $50 \%$ of its length.

The angles $\zeta$ and $\eta$ have no physical significance because, when bending a beam, there is almost only deformation in the $O x y$ plane. The $\xi$ curve (Fig. 6) represent the angle made by the beam through its length. Both the deflection and the Eulerian angle curves match perfectly for both methods. Consequently, both methods lead to results with good precision. Although the polar decomposition method has a stronger mathematical definition, the computer effort required for calculation with this algorithm is greater due to the need for more steps, when compared to the incremental rotation method. This computer effort becomes larger because it must be performed twice for each integration point and for each stress increment when a mid-increment scheme is adopted [40].

\section{Concluding remarks}

Plastic behaviour of metals is influenced not only by the amount of plastic strain, but also by the strain rate and temperature at which strain occurs or is induced [33]. Strain rate history and temperature history effects become evident in experiments in which temperature is changed during the deformation process.

Using a relatively simple set of equations it is possible to model the plastic deformation of a material over a wide range of temperatures.

The relations described on Section 3.1 provide an approximate set of constitutive equations which may be used to study material behaviour from room temperature up to hot-working temperature. 
The calculations of the rotation tensor were made by the polar decomposition and the rotation increment methods. Both methods revealed suitable to solve the problems discussed in this work.

The model characterizes fairly well real material behaviour and duplicates the experimental results obtained with different solicitations and temperatures.

The material parameters necessary for the constitutive model were determined fitting experimental data obtained by the authors. Elevated temperature experiments are currently being performed to further refine the constitutive equations and the constitutive equation parameters.

\section{Acknowledgments}

The authors acknowledge the financial support given by the FCT_-Portuguese Foundation for Science and Technology and by the Programa Operacional Ciência, Tecnologia, Inovação do Quadro Comunitário de Apoio III, FEDER.

\section{References}

[1] H.L. Xing, A. Makinouchi, 3-D Thermal-elastic-plastic in finite deformation and its application to non-isothermal sheet forming, in: D.R.J. Owen, E. Onate, E. Hinton (Eds.), Computational Plasticity_Fundamentals and Applications, vol. 2, CIMNE, Barcelona, 1997 , pp. $1443-1452$.

[2] L. Anand, A. Lush, M.F. Briceno, D.M. Parks. A time-integration procedure for a set of internal variable type elasto-viscoplastic constitutive equations, Research Report-Department of Mechanical Engineering, MIT, USA, 1985.

[3] L. Anand, Constitutive equations for hot-working of metals, Int. J. Plasticity 1 (1985) 213-231.

[4] C. Teodosiu, P. Genevois, Modelling large deformation anisotropic plastic behaviour of mild steel sheet, in: Proceedings of the International symposium MECAMAT on inelastic behaviour of solids: models and utilisation, Besanon, France, 1988, pp. 211-225.

[5] L.F. Menezes, Modelação tridimensional e simulação numérica dos processos de enformação por deformação plástica, Ph.D. Thesis, Universidade de Coimbra, Portugal, 1994.

[6] C.A. Filippa, A systematic approach to the element-independent corotational dynamics of finite elements, Report CU-CAS-00-03, Department of Aerospace Engineering Sciences and Center for Aerospace Structures, University of Colorado, USA, 2000.

[7] J.C. Simo, T.J.R. Hughes, Computational Inelasticity, Springer-Verlag Inc., New York, 1998.

[8] M. Ortiz, Some computational aspects of finite deformation plasticity, in: D.R.J. Owen, E. Hinton, E. Oñate (Eds.), Proceedings of the 2nd International Conference on Computational Plasticity, Barcelona, Spain, 1987, pp. 1717-1756.

[9] A.E. Green, P.M. Naghdi, A general theory of an elastic plastic continuum, Arch. Ration. Mech. Anal. 18 (1965) $251-281$.

[10] A.E. Green, P.M. Naghdi, Some remarks on elastic-plastic deformations at finite strains, Int. J. Engrg. Sci. 9 (1971) $1219-1229$.

[11] R.M. McMeeking, J.R. Rice, Finite-element formulation for problems of large elastic-plastic deformation, Int. J. Solids Struct. 2 (1975) 601-616.

[12] J.D. Nagtegaal, J.E. DeJong, Some computational aspects of elastic-plastic large strain analysis, Int. J. Numer. Methods Engrg. 17 (1981) $15-41$.

[13] F. Sidoroff, Formulation elasto-plastique en grandes déformations, Rapport GRECO 29, 1981.

[14] F. Sidoroff, Incremental constitutive equation for large strain elasto-plasticity, Int. J. Engrg. Sci. 20 (1982) 19-38.

[15] J. Rice, Numerical Methods, Software, and Analysis, International Student Edition, McGraw-Hill, 1998.

[16] A. Gullerud, K. Koppenhoefer, A. Roy, S. Chowdhury, M. Walters, R.H. Dodds Jr., WARP3D—3D Dynamic nonlinear fracture analysis of solids using parallel computers and workstations, Report on a Research Project sponsored by the US Nuclear Regulatory Commission and The NASA Ames Research Center, Civ. Dep., University of Illinois, USA, 2002.

[17] J.P. Ponthot, Unified stress update algorithms for the numerical simulation of large deformation elasto-plastic and elasto-viscoplastic processes, Int. J. Plasticity 18 (2002) 91-126.

[18] P.A. Dashner, Invariance considerations in large strain elasto-plasticity, J. Appl. Mech. 53 (1986) 55-66.

[19] C. Teodosiu, The plastic spin: microstructural origin and computational significance, in: D.R.J. Owen, E. Hinton, E. Oñate (Eds.), Proceedings of the 2nd International Conference on Computational Plasticity, Barcelona, Spain, 1989, pp. 163-175.

[20] G.C. Johnson, D.J. Bammann, A discussion of stress rates in finite deformation rates in finite deformation problems, Int. J. Solids Struct. 20 (1984) $725-737$.

[21] A. Dogui, Cinématique bidimensionelle en grandes deformations - application la traction hors axes et la torsion, Rap. GRECO - Grandes déformations et endommagement, Ecole Centrale de Lyon, France, 1987.

[22] Y.F. Dafalias, Corotational rates for kinematic hardening at large plastic deformations, J. Appl. Mech. 50 (1983) $561-565$.

[23] S. Roy, A.F. Fossum, R.J. Dexter, On the use of polar decomposition in the integration of hypo-elastic constitutive laws, Int. J. Engrg. Sci. 30 (1992) $119-133$.

[24] J.P. Ponthot, Traitement unifié de la mécanique des milieux continus solides en grandes transformations par la méthode des éléments finis, Ph.D. Thesis, Université de Liege, Belgium, 1995.

[25] T. Hughes, Numerical implementation of constitutive models: rate-independent deviatoric plasticity, in: S. Nemat-Nasser, R. Asaro, G. Hegemier (Eds.), Theoretical Foundation for Large-scale Computations of Nonlinear Material Behaviour, Martinus Nijhoff, 1983.

[26] S.B. Brown, H.K. Kwon, L. Anand, An internal variable constitutive model for hot working of metals, Int. J. Plasticity 5 (1989) $95-130$.

[27] F. Teixeira-Dias, L.F. Menezes, Numerical aspects of finite element simulations of residual stresses in metal matrix composites, Int. J. Numer. Methods Engrg. 50 (2001) 629-644.

[28] H.L. Xing, A. Makinouchi, Three dimension finite element modeling of thermomechanical frictional contact between finite deformation bodies using $R$-minimum strategy, Comput. Methods Appl. Mech. Engrg. 191 (2002) 4193-4214.

[29] M. Kleiber, Computational coupled non-associative thermo-plasticity, Comput. Methods Appl. Mech. Engrg. 90 (1991) $943-967$.

[30] F. Teixeira-Dias, L.F. Menezes, A kinematic and incremental integration model for the micromechanical numerical analysis of dual-phase materials, Comput. Mater. Sci. 25 (2002) 237-245.

[31] F. Teixeira-Dias, L.F. Menezes, Numerical determination of the influence of the cooling rate and reinforcement volume fraction on the levels of residual stresses in Al-SiC composites, Comput. Mater. Sci. 21 (2001) 26-36. 
[32] A.K. Miller, O.D. Sherby, A simplified phenomenological model for non-elastic deformation: prediction of pure aluminum behavior and incorporation of solute strengthening effects, Acta Metall. 26 (1978) 289-304.

[33] S. Balasubramaniam, L. Anand, Elasto-viscoplastic constitutive equations for polycrystalline fcc materials at low homologous temperatures, J. Mech. Phys. Solids 50 (2002) 101-126.

[34] R.D. Krieg, S.W. Key, in: J.A. Striccklin, K.J. Saczalsky (Eds.), Implementation of a Time Independent Plasticity Theory into Structural Computer Programs, Constitutive Equations in Viscoplaticity: Computational and Engineering Aspects, AMD-20, ASME, New York, USA, 1976 , pp. 125-138.

[35] A. Lush, L. Anand, Implicit time-integration procedures for a set of internal variable constitutive equations for hot-working, in: Proceedings of the NUMIFORM'86, Gothenburg, Germany, 1986, pp. 131-137.

[36] A.M. Lush, G. Weber, L. Anand, An implicit time-integration procedure for a set of internal variable constitutive equations for isotropic elastoviscoplasticity, Int. J. Plasticity 5 (1989) 521-549.

[37] A. Hoger, D.E. Carlson, Determination of the stretch and rotation in the polar decomposition of the deformation gradient, Quart. Appl. Math. 42 (1984) 113-117.

[38] T.C.T. Ting, Determination of C1/2, C-1/2 and more general isotropic tensor functions of C, J. Elasticity 15 (1985) $319-323$.

[39] H.L. Cao, Modélisation mecanique et simulation numérique de l'emboutissage, Ph.D. Thesis, INPG-Grenoble, France, 1990.

[40] T.J.R. Hughes, J. Winget, Finite rotation effects in numerical integration of rate constitutive equations arising in large-deformation analysis, Int. J. Numer. Methods Engrg. 15 (1980) 1862-1867.

[41] A.B. Lopes, F. Barlat, J.J. Gracio, J.F. Ferreira Duarte, E.F. Rauch, Effect of texture and microstructure on strain hardening for aluminum deformed in uniaxial tension ad simple shear, Int. J. Plasticity 19 (2003) 1-22.

[42] A. Andrade-Campos, L.F. Menezes, F. Teixeira-Dias, Large deformation processes on AA1050-O aluminium at elevated temperatures, in: Proceedings of the II International Materials Symposium, Materiais 2003, FCT/UNL, Caparica, Portugal, vol. 1, 2003 , p. 251.

[43] T.J.R. Hughes, Generalization of selective integration procedures to anisotropic and non-linear media, Int. J. Numer. Methods Engrg. 15 (1980) 1413-1418.

[44] P.M. Pinsky, M. Ortiz, K.S. Pister, Numerical integration of rate constitutive equations in finite deformation analysis, Comput. Methods Appl. Mech. Engrg. 12 (1983) 353-364.

[45] O.C. Zienkiewicz, R.L. Taylor, fifth ed The Finite Element Method, vol. I, Butterworth-Heinemann, 2001.

[46] T. Nakamura, C. Shih, L. Freund, Analysis of dynamically loaded SE(B) ductile fracture specimen, Engrg. Fract. Mech. 25 (1986) $323-339$.

[47] T. Belytschko, W.K. Liu, B. Moran, Nonlinear Finite Elements for Continua Structures, John Wiley \& Sons, Ltd, West Sussex, England, 2000.

[48] J.C. Nagtegaal, D.M. Parks, J.R. Rice, On numerically accurate finite element solutions in the fully plastic range, Comput. Methods Appl. Mech. Engrg. 4 (1974) 153

[49] H. Haddadi, Matériaux composites à matrice métallique: modélisation du comportement thermoélastiqueviscoplastique et résolution de problèmes aux limites 3D par la méthode finis, Ph.D. Thesis, Laboratoire des Propriétés Mécaniques et Thermodynamics des Matériaux, Université Paris-Nord, 1996.

[50] A. Andrade-Campos, L.F. Menezes, F. Teixeira-Dias, On the numerical analysis of rate-dependent large deformation processes at elevated temperatures, in: D.R.J. Owen, E. Onate, B. Suárez (Eds.), Computational Plasticity VII-Fundamentals and Applications, 2003 , p. 189. 